\title{
Biomarkers for Alzheimer's disease: the dilemma of silent disease
}

Alzheimer's disease researchers have homed in on a few molecules in the cerebrospinal fluid (CSF) that may help diagnose Alzheimer's disease before clear-cut symptoms emerge. Niklas Mattsson et al. ${ }^{1}$ have now conducted the largest trial of such biomarkers in 1,583 subjects-including 750 people with mild cognitive impairment who were followed for at least two years after measurement of the markers. They found disparate outcomes among the various trial sites, highlighting the need to better standardize procedures. They also showed that the molecules could predict incipient Alzheimer's disease with what they called "good accuracy."

\section{Elaine R Peskind:}

The study illuminates some fundamental problems in the field. Employing clinical diagnoses as the gold standard to which CSF biomarkers are compared introduces several sources of misclassification which reduce their validity. Such sources include misdiagnosis, combination underlying pathologies and, probably most importantly, latent or 'silent' pathological changes of Alzheimer's disease among cognitively normal persons. Autopsy series as well as longitudinal CSF biomarker studies suggest that true preclinical disease with progressive brain pathology may proceed for as long as a decade or more in the absence of any clinical signs or symptoms of dementia in a substantial number of older cognitively normal persons. Thus, CSF amyloid- $\beta 42\left(A \beta_{42}\right)$, tau and $p$-tau ${ }_{181}$ concentrations may be better indicators of Alzheimer's disease pathology than these types of studies suggest.

Associate Director, University of Washington Alzheimer's Disease Research Center, Seattle, Washington, USA.

\section{David Holtzman:}

Many studies have shown that CSF levels of $A \beta_{42}$ are decreased in indviduals with Alzheimer's disease and that levels of forms of tau are increased. These molecules alone and, even more so, together, are strongly predictive of the conversion of individuals with mild cognitive impairment (MCI) to clearcut Alzheimer's disease-type dementia. The same markers are also predictive of conversion from cognitive normality to MCI. As in smaller, single-site studies, Mattsson et al. ${ }^{1}$ studied over 1,500 individuals and found that CSF $A \beta_{42}$ and forms of tau were predictive of conversion from MCI to Alzheimer's disease both alone and, to a greater extent, together. Although this study points out technical issues that are important to address in future studies, these data confirm the usefulness of these markers in identifying individuals for entry into clinical trials earlier in the course of the Alzheimer's disease than has been studied in the past. It's time to use CSF A $\beta_{42}$, tau and p-tau in all Alzheimer's trials.

Chair, Washington University School of Medicine Department of Neurology, St. Louis, Missouri, USA.

\section{COMPETING INTERESTS STATEMENT}

The author declares competing financial interests: details accompany the full-text HTML version of the paper at http://www.nature.com/ naturemedicine/.
It's time to use CSF $A \beta_{42}$, tau and p-tau in all Alzheimer's trials. —David Holtzman

\section{Leslie Shaw:}

Approaches that combine standardized CSF biomarkers with other standardized measures of disease may provide the best strategy for detection of the often complex pathology of incipient Alzheimer's disease and its time course in individuals with MCI. Large-scale investigations of longitudinal progression of incipient Alzheimer's disease-using standardized clinical evaluations, biofluid sample collection procedures, CSF analyses and imaging modalities - are well underway. Results from such studies confirm and extend the findings, both by Mattsson et al. ${ }^{1}$ and by earlier single-center studies, of detection of Alzheimer's disease pathology in individuals with MCI. These standardized approaches for biomarker measurements are ready for incorporation into clinical trials.

Incorporation of independently developed cutpoints to help assign MCI study subjects to appropriate risk groups, such as high risk for Alzheimer's disease and low risk for Alzheimer's disease, is also essential.

Results from multicenter studies such as the Alzheimer's Disease Neuroimaging Initiative are actively testing the hypothesis that a combination of well-standardized CSF biomarkers and imaging biomarkers provides the overall best approach to the most accurate detection of incipient Alzheimer's disease and characterization of disease progression.

Important research initiatives are also underway for discovery and validation of new CSF and plasma biomarkers. These new biomarkers detect other pathways involved in Alzheimer's disease pathology or other misfolded proteins that reflect pathologic lesions such as Lewy bodies. These investigations test the hypothesis that most detailed characterization of incipient Alzheimer's disease and discrimination from other dementias can be improved by combining new CSF biomarkers with the established ones described by Mattsson et al. ${ }^{1}$. If successful, such studies could lead to even better diagnostic accuracy than that now achieved.

Professor, Department of Pathology and Lab Medicine,

University of Pennsylvania School of Medicine, Philadelphia, Pennsylvania, USA.

1. Mattsson, N. et al. CSF biomarkers and incipient Alzheimer's disease in patients with mild cognitive impairment. J. Am. Med. Assoc. 302, 385-393 (2009). 